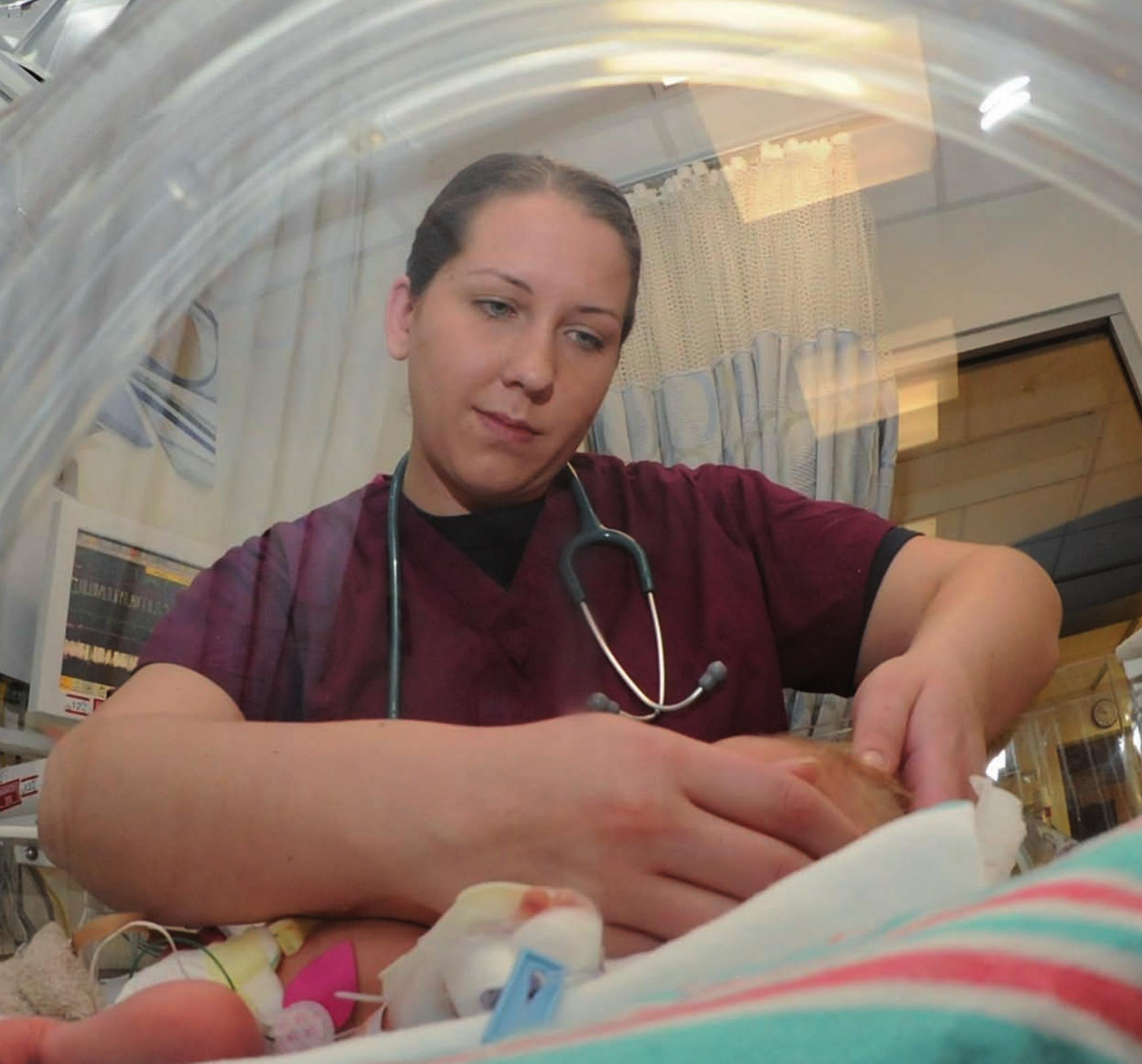




\section{Newborn screening for cystic fibrosis: pros and cons}

\section{Educational aims}

1 To give an insight into the arguments that have led to the implementation of newborn screening for cystic fibrosis in routine screening programmes.

1 To understand the drawbacks specifically associated with newborn screening for cystic fibrosis and how these can be handled or avoided.

\section{Summary}

During the past decade, newborn screening for cystic fibrosis has been introduced in many countries. However, the disadvantages associated with newborn screening for CF hamper its introduction in other areas.

Within the routine heel-prick programmes implemented around the world, there is no other disease that elicited such a debate about whether or not to include it in these programmes as cystic fibrosis (CF).

The reasons for this debate are manifold: the diagnosis of CF is not as simple as it looks on first sight; a robust screening test suitable for universal use does not exist; and the effects of early management on the outcome are doubted by many. The improvement in survival of patients with CF observed during the last few decades [1] has been attributed to an improvement of the traditional therapies for CF. Examples include: pancreatic enzyme replacement therapy, high caloric intake and supplementation of fatsoluble vitamins for CF gastrointestinal disease; early eradication of airway infection with Pseudomonas aeruginosa (inhalation therapy with antibiotics when the infection becomes chronic); and improved therapies for mucociliary clearance using dornase alfa and hypertonic saline. However, therapies aimed at the basic defect have not yet been developed.

Newborn screening for CF allows early diagnosis, often when the child is still asymptomatic, or only has a few symptoms related to poor growth but no pulmonary problems. However, the evidence that starting treatment before respiratory problems have arisen leads to a better outcome on the long term is not very strong, but there is ample evidence that in the first decade of life, CF patients identified by screening have a better clinical condition than patients diagnosed clinically.

In this article we shall discuss the most common arguments for and against newborn screening for CF (NBSCF).

\section{Arguments for NBSCF}

The best arguments for screening can be found when observing cohorts of patients with CF diagnosed clinically in areas where no newborn screening programme for $\mathrm{CF}$ is running.

Newborn screening leads to diagnosis at a very young age. In most countries without newborn screening, the median age of diagnosis is $\sim 1 \mathrm{yr}$, and when patients with a meconium ileus are excluded, the median age is even higher; for example, in Canada, the
J.E. Dankert-Roelse

A. Vernooij-van Langen

Dept of Pediatrics, Atrium Medisch Centrum, Heerlen, The Netherlands

Correspondence

J.E. Dankert-Roelse

Atrium Medisch Centrum

Parkstad - Paediatrics

Henri Dunantstraat 5

P0Box 4446 Heerlen 6401 CX

The Netherlands

jdankert@atriummc.nl

Competing interests

None declared.

HERMES syllabus link: module B.15.1 
median age at diagnosis is $>2$ yrs and in Sweden, it is $\sim 6$ months $[2,3]$, while with a newborn screening programme, the median age at diagnosis decreases to 29 days [4].

In regions without screening, clinical symptoms finally lead to the diagnosis. Observational data from Canada show that $81 \%$ of clinically diagnosed CF patients have respiratory symptoms, many show failure to thrive $(37 \%)$ and reduced serum concentrations of fatsoluble vitamins due to malabsorption, and 95\% have gastrointestinal complaints at the time of diagnosis [2]. The mean head circumference of patients with a vitamin E deficiency was significantly lower, at the 32nd percentile $(95 \% \mathrm{Cl}$ 24-41\%), than that of patients with a normal vitamin $\mathrm{E}$ concentration, of whom the mean head circumference was at the 63th percentile (95\% Cl 47-78\%) [2].

Hospital admissions are not uncommon during the period in which children develop symptoms: $79 \%$ of the children were hospitalised, with a mean length of stay of 5 days (range 1-30 days) in a tertiary care hospital before the diagnosis CF was made. Some children already had severe bronchiectasis at the time of diagnosis [5].

Many of these problems associated with a late diagnosis can be prevented. Evidence for beneficial effects of screening has been reported in two randomised clinical trials [6-8], in analyses form large clinical databases [9-11] and from a few cohort studies [12-17].

\section{Treatment of pancreatic insufficiency}

Gastrointestinal problems in CF are mostly related to pancreatic insufficiency. Most patients with CF are pancreatic insufficient at birth or develop pancreatic insufficiency during the first year of life [18]. With an early diagnosis, pancreatic disease can be treated from the start with pancreatic enzyme replacement therapy.

\section{Growth}

In a randomised clinical trials $[6,8]$, a large clinical database [9] and four observational cohort studies, a significant improvement of height and weight was observed compared with clinically diagnosed CF $[11,14,16,17]$. With an early diagnosis, children with CF can maintain a growth pattern that approximates that of healthy children [6].

\section{Nutritional deficiencies}

Malnutrition by protein deficiency and significant morbidity due to deficiencies of fat-soluble vitamins have been described in clinically diagnosed patients $[19,20]$, and newborn screening can prevent this morbidity. Vitamin deficiency can occur very early in life. Many infants diagnosed by screening already show low concentrations of vitamin A (60\%), D $(37 \%)$ and $E(16 \%)[21]$, underlining the need for early diagnosis and start of treatment.

There is indirect evidence that early malnutrition and nutritional deficiencies early in life are related to lung growth. Patients with CF with pancreatic insufficiency who achieved early growth recovery within 2 years of diagnosis had fewer cough symptoms, higher lung function and better chest radiography scores at 6 years of age [22].

\section{Lung function}

A randomised clinical trial showed better chest radiography scores early in life but at a later age, no difference in lung function between the screened cohort and the clinically diagnosed patients was found [23]. This may be related to the fact that the screened cohort had earlier P. aeruginosa colonisation than the clinically diagnosed cohort. In all cohort studies, significantly better chest radiography scores were observed in the screened cohorts. Crosssectional studies with data derived from the US database found better lung function in screened patients aged 6-10 and 1120 years [20]. Cohort studies from the Netherlands and Australia showed better lung function until 12 and 15 years of age in the screened cohorts, respectively $[12,15]$. In the UK database, no differences in lung function was observed between screened and nonscreened cohorts, but the screened cohort needed significantly less treatment [24].

There is also indirect evidence that early diagnosis and treatment are related to better lung function, as a cohort study showed that in sibling pairs, the younger siblings show a significantly better lung function at adult age [25]. Moreover, while recruiting patients $<12$ years of age for the Early Pseudomonas Infection Control (EPIC) study, it was found that patients with a diagnosis through newborn screening had a significantly better forced expiratory volume in $1 \mathrm{~s} \%$ predicted than clinically diagnosed patients [26]. 
It is possible to eradicate $P$. aeruginosa during the first infection in practically all patients, but practically impossible to eradicate $P$. aeruginosa when the infection has become chronic. Pseudomonas can colonise the airways of young infants with CF in the first months of life without causing symptoms but leading to infection, inflammation and structural lung disease [27]. The observation that an early diagnosis does not change the time to first acquisition of Pseudomonas is another argument for the need for NBSCF: with an early diagnosis and thorough monitoring of airway infection, it is possible to start eradication treatment of the first infections with pathogenic micro-organisms, which can postpone chronic infection for a long time.

\section{Survival}

In one randomised clinical trial, a higher risk was found for an early death in the clinically diagnosed patients [7]; a systematic review also reported a lower mortality risk in screened cohorts [28].

\section{Hospital admissions and burden of care}

Fewer hospital admissions were found in one randomised clinical trial [7] and in two cohort studies $[16,20]$. In the UK database cohort study, less therapy was needed, and improved growth and reduced morbidity were found in the screened cohort [24].

\section{The opinion of parents}

Parents prefer an early diagnosis even when their child has an untreatable disorder [29] in order to prevent a long diagnostic odyssey. In a recent study in Sweden, where currently no NBSCF takes place, parents favoured screening [30]. Parents of children with CF with a diagnosis within the first 3 months of life had more confidence in the medical profession and less negative feelings than parents of children with a late CF diagnosis [31].

An early diagnosis of a genetic disease such as CF also renders the opportunity that parents receive genetic counselling, which is important for further family planning. In some countries with longstanding newborn screening programmes for $\mathrm{CF}$, the prevalence of $\mathrm{CF}$ at birth has been steadily diminished during the last 20 years [32].

\section{Research needs}

Present treatment for CF cannot reduce the increased susceptibility of CF airways to bacterial infection, and once the airways have become infected it is extremely difficult to eradicate the invading micro-organisms. There is a need for research that can elucidate the process of bacterial infection in the CF airway, which may lead to improved treatment options. Clinical trials that investigate interventions in young infants without pulmonary involvement can only be performed when infants with CF are identified early in life, before pulmonary involvement, i.e. by newborn screening.

\section{Economic arguments}

Newborn screening for CF seems cost effective and can lead to cost savings, as costs of treatment can be reduced by $10 \%[33,34]$. A more recent analysis assumed that the costs of screening, diagnosis and treatment in the first 3 years of life are about $71 \%$ of the costs of diagnosis and treatment without a newborn screening programme, which means that NBSCF can lead to savings [35].

\section{Arguments against NBSCF}

\section{Effect on parents due to abnormal results of newborn screening tests}

Most parents experience high levels of emotional stress during their wait for further diagnostic testing, mostly the sweat test. It may cause depressive symptoms that vary depending on their perceptions of how likely it is their child has CF [36]. A good protocol for handling abnormal screening test results with a minimum waiting period between the notification of the abnormal test result and the sweat test, and good information of the parents may reduce much of the parental stress [37].

\section{False-positive tests}

Practically all screening programmes lead to abnormal results that, during the following diagnostic process, appear to have been a false alarm. Such false alarms often lead to parental stress and anxiety. However, in the long-term, when the child appears to be healthy and with good parental education, 
parental anxiety levels do not differ from parents of children who did not get such an alarm [37].

\section{Identification of carriers}

Screening approaches that include CF transmembrane conductance regulator (CFTR) mutation analysis also identify healthy infants carrying one CFTR mutation. An advantage of this finding may be that parents are offered genetic counselling, and occasionally both parents may turn out to be carriers. This can be important information for further family planning. However, in most cases, only one parent will be identified as a carrier, which can lead to anxiety and stress. From the child's perspective, the knowledge of being a carrier is not of direct and immediate benefit. Moreover, as the child could not decide whether or not he/she wished to be tested, this can be considered as a violation of the "right not to know".

\section{Inconclusive results of NBSCF}

In most screening programmes, quite a number of infants are found by newborn screening in whom the diagnosis CF cannot be confirmed nor excluded, because they do not meet the diagnostic criteria for CF. Mostly, they have elevated immunoreactive trypsinogen (IRT) concentrations, normal or borderline sweat chloride concentrations, and carry one or two CFTR mutations with an unclear or unknown pathogenic effect on phenotype. Some of these infants will develop a complete or partial CF phenotype later in life, but many will never develop any symptoms. The uncertainty about prognosis is difficult for genetic counselling and requires that such infants get a regular further follow-up at CF centres. This may have a lifelong impact on the child and his or her family.

\section{Risk for early colonisation with $P$. aeruginosa}

Various studies showed less chronic airway infection with $P$. aeruginosa [10, 13, 20, 24]. However, in one randomised clinical trial, infants identified by screening developed colonisation and chronic infection with $P$. aeruginosa at an earlier age than clinically diagnosed patients with CF [23]. This may relate to crossinfection. The finding underlined the fact that patients with CF have an innate increased susceptibility for early airway colonisation with opportunistic micro-organisms, such as
Staphylococcus aureus and P. aeruginosa. Infants identified by newborn screening will mostly visit CF clinics at an earlier age than clinically diagnosed patients; special hygienic measures to prevent cross-infections from older CF patients should be in place in every CF clinic.

\section{Risk of ethnic discrimination}

The use of a screening strategy including CFTR mutation analysis carries the risk that very rare mutations will not be identified. In countries and cities with large multiethnic populations, this may lead to more missed cases in infants originating from non-Western countries. A European survey of mutations in CF patients of NorthAfrican or Turkish descent showed that only 63\% of patients of Turkish origin would be identified by newborn screening [38]. It may be possible to compensate by using procedures such as a second IRT test if the first level was very high, or by performing DNA scanning of the CFTR gene in samples with very high IRT concentrations without mutations in the common panel.

Many of the hazards associated with running a newborn screening programme for CF can be reduced. Notification of abnormal test results should lead to prompt referral and diagnostic testing according to an established protocol with solid parental information. Parents should be directed to safe and reliable sources of information on internet. Novel screening strategies can drastically reduce the number of false positives and equivocal diagnosis [35]. Infants with a CF diagnosis should not be exposed to older patients with CF to prevent cross-infection with P. aeruginosa.

\section{Discussion}

Most arguments that favour NBSCF seem to be dependent on the management and treatment that can be given to the child with CF identified by screening. NBSCF leads to diagnosis before lung disease and malnutrition have appeared. Whilst after a clinical diagnosis, all efforts are primarily directed to treating disease in the affected organs, after a diagnosis by newborn screening, the main challenge is to keep the infant as healthy as possible. This should lead, and often does lead, to a change to proactive, preventive medicine rather than reactive damage limitation. Recent evidence supports the notion that very early treatment of pulmonary infection and inflammation is a key factor for further improving prognosis of patients with CF 
and further research in this area is urgently needed.

Most arguments against screening seem to be related to the imperfect properties of the available screening tests for $\mathrm{CF}_{\text {; }}$ therefore, as tests improve, most of the arguments against screening will disappear. Since May 2011, the Dutch newborn screening programme has been extended with screening for CF after a pilot study assessing the characteristics of two novel screening strategies $[35,39]$. The screening test now in use in the Netherlands differs from screening tests used in other countries: it has a very high specificity and positive predictive value, and detects only a very small number of carriers and patients with an equivocal diagnosis [35]. With this screening programme, most of the arguments against screening, as discussed in this article, are no longer relevant.
Finally, costs of screening cannot be used as an argument against newborn screening for CF. Although the savings of an early diagnosis of CF are not as clear cut as in newborn screening for congenital hypothyroidism or phenylketonuria, NBSCF and treatment appear to cost less than a clinical diagnosis and treatment [25].

\section{Conclusion}

The benefits of NBSCF are now generally accepted. The benefits of NBSCF amply compensate for the disadvantages. Moreover, NBSCF is costeffective and there is growing evidence that it leads to cost savings. Many of the disadvantages of NBSCF are reduced to practically nil with the novel screening strategy that has recently been introduced in the Netherlands.

\section{References}

1. Dodge JA, Lewis PA, Stanton M, et al. Cystic fibrosis mortality and survival in the UK: 1947-2003. Eur Respir J 2007; 29: 522-526.

2. Steinraths M, Vallance HD, Davidson AGF. Delays in diagnosing cystic fibrosis. Can Fam Physician 2008; 54: 877-873.

3. De Monestrol I, Klint A, Sparén P, et al. Age at diagnosis and disease progression of cystic fibrosis in an area without newborn screening. Paediatr Perinat Epidemiol 2011; 25: 298-305.

4. Comeau AM, Parad RB, Dorkin HL, et al. Population-based newborn screening for genetic disorders when multiple mutation DNA testing is incorporated: a cystic fibrosis newborn screening model demonstrating increased sensitivity but more carrier detections. Pediatrics 2004; 113: 1573-1581.

5. Dankert-Roelse JE, Mérelle ME, Laarman AR, et al. [Young children with serious disorders as a result of late diagnosis of cystic fibrosis]. Ned Tijdschr Geneeskd 2006; 150: 525-529.

6. Farrell PM, Lai HJ, Li Z, et al. Early diagnosis of cystic fibrosis through neonatal screening prevents severe malnutrition and improves long-term growth. Wisconsin Cystic Fibrosis Neonatal Screening Study Group. Pediatrics 2001; 107: 1-13.

7. Doull IJ, Ryley HC, Weller P, et al. Cystic fibrosis-related deaths in infancy and the effect of newborn screening. Pediatr Pulmonology 2001; 31: 363-366.

8. Southern KW, Mérelle MM, Dankert-Roelse JE, et al. Newborn screening for cystic fibrosis. Cochrane Database Syst Rev 2009; 1: CD001402.

9. Wang SS, $0^{\prime}$ Leary LA, Fitzsimmons SC, et al. The impact of early cystic fibrosis diagnosis on pulmonary function in children. J Pediatr 2002; 141: 804-810.

10. Lai HJ, Cheng $\mathrm{Y}$, Cho $\mathrm{H}$, et al. Association between initial disease presentation, lung disease outcomes, and survival in patients with cystic fibrosis. Am J Epidemiol 2004; 159: 537-546.

11. Sims EJ, Clark A, McCormick J, et al. Cystic fibrosis diagnosed after 2 months of age leads to worse outcomes and requires more therapy. Pediatrics 2007; 109: 19-28.

12. Mérelle ME, Schouten JP, Gerritsen J, et al. Influence of neonatal screening and centralized treatment on long-term clinical outcome and survival of CF patients. Eur Respir J 2001; 18: 306-315.

13. Dankert-Roelse JE, Mérelle ME. Newborn screening for CF: published evidence from Europe. J Pediatrics 2005; 147: Suppl., S15-S20.

14. Waters DL, Wilcken B. Irwing L, et al. Clinical outcomes of newborn screening for cystic fibrosis. Arch Dis Child Fetal Neonatal Ed 1999; 80: F1-F7.

15. McKay K0, Waters DL, Gaskin KJ. The influence of newborn screening for cystic fibrosis on pulmonary outcomes in New South Wales. J Pediatr 2005; 147: Suppl., S47-S50.

16. Siret D, Bretaudeau G, Branger B, et al. Comparing the clinical evolution of cystic fibrosis screened neonatally to that of cystic fibrosis diagnosed from clinical symptoms: a 10-year retrospective study in a French region (Brittany). Ped Pulmonol 2003; 35: 342-349.

17. Mastella G, Zanolla L, Castellani C, et al. Neonatal screening for cystic fibrosis: long-term balance. Pancreatology 2001; 1: 531-537.

18. Waters DL, Dorney SFA, Gaskin KJ, et al. Pancreatic function in infants identified as having cystic fibrosis in a neonatal screening program. N Eng J Med 1990; 322: 303-308.

19. Mérelle ME, Griffioen RW, Dankert-Roelse JE. Cystic fibrosis presenting with intracerebral haemorrhage. Lancet 2001; 358: 1960.

20. Accurso FJ, Sontag MK, Wagener JS. Complications associated with symptomatic diagnosis in infants with cystic fibrosis. J Pediatr 2005; 147: Suppl., S37-S41.

21. Neville LA, Ranganathan SC. Vitamin D in infants with cystic fibrosis diagnosed by newborn screening. J Paediatr Child Health 2009; 45: 36-41.

22. Lai HJ, Shoff SM, Farrell PM, et al. Recovery of birth weight z score within 2 years of diagnosis is positively associated with pulmonary status at 6 years of age in children with cystic fibrosis. Pediatrics 2009; 123: 714-722. 
23. Farrell PM, Li Z, Kosorok MR, et al. Bronchopulmonary disease in children with cystic fibrosis after early or delayed diagnosis. Am J Respir Crit Care Med 2003; 168: 1100-1108.

24. Sims EJ, Mugford M, Clarj A, et al. Economic implications of newborn screening for cystic fibrosis: a cost of illness retrospective cohorts study. Lancet 2007; 7: 1187-1195.

25. Slieker MG, van den Berg JMW, Kouwenberg J, et al. Long-term effects of birth order and age of diagnosis in cystic fibrosis: a sibling cohort study. Pediatr Pulmonol 2010; 45: 601-607.

26. Rosenfeld M, Emerson J, McNamara S, et al. Baseline characteristics and factors associated with nutritional and pulmonary status at enrollment in the cystic fibrosis EPIC observational cohort. Ped Pulmonology 2010; 45: 934-944.

27. Sly PD, Brennan S, Gangell C, et al. Lung disease at diagnosis in infants with cystic fibrosis detected by newborn screening. Am J Respir Crit Care Med 2009; 180: 146-150.

28. Grosse SD, Rosenfeld M, Devine 0J, et al. Potential impact of newborn screening for cystic fibrosis on child survival: a systematic review and analysis. J Pediatr 2006; 149: 362-366.

29. Plass AM, van El CG, Pieters T, et al. Neonatal screening for treatable and untreatable disorders: prospective parents' opinions. Pediatrics 2010; 125: e99-e106.

30. De Monestrol I, Brucefors AB, Sjöberg B, et al. Parental support for newborn screening for cystic fibrosis. Acta Paediatrica 2011; 100: 209-215.

31. Mérelle ME, Huisman J, Alderden-van der Vecht A, et al. Early versus late diagnosis: psychological impact on parents of children with cystic fibrosis. Pediatrics 2003; 111: 346-350.

32. Sims EJ, Mugford M, Clark A, et al. Economic implications of newborn screening for cystic fibrosis: a cost of illness retrospective cohort study. Lancet 2007; 7: 1187-1195.

33. Van den Akker-van Marle ME, Dankert HM, Verkerk PH, et al. Cost-effectiveness of 4 neonatal screening strategies for cystic fibrosis. Pediatrics 2006; 118: 896-905.

34. Tluczek A, Koscik RL, Farrell PM, et al. Psychosocial risk associated with newborn screening for cystic fibrosis: parents' experience while awaiting the sweat-test appointment. Pediatrics 2005; 115: 1692-1703.

35. Health Council of the Netherlands. Neonatal Screening for Cystic Fibrosis, The Hague, Health Council of the Netherlands, 2010.

36. Vernooij-van Langen AM, Reijntjens SJ, van der Pal SM, et al. Do false positive test results for newborn screening for cystic fibrosis lead to long term parental anxiety? J Cyst Fibros 2011; 10: S11.

37. Lakeman P, Gille JJ, Dankert-Roelse JE, et al. CFTR mutations in Turkish and North African cystic fibrosis patients in Europe: implications for screening. Genet Test 2008; 12: 25-35.

38. Massie J, Curnow L, Gaffney L, et al. Declining prevalence of cystic fibrosis since the introduction of newborn screening. Arch Dis Child 2010; 95: 531-533. 